Musées, Patrimoine et Culture scientifiques et techniques

$191 \mid 2020$

septembre-octobre 2020

\title{
Dans la jungle des formations
}

\section{Serge Chaumier}

URL : https://journals.openedition.org/ocim/4076

DOI : $10.4000 /$ ocim.4076

ISSN : 2108-646X

Éditeur

OCIM

Édition imprimée

Date de publication : 1 septembre 2020

Pagination : 22-29

ISSN : 0994-1908

Référence électronique

Serge Chaumier, « Dans la jungle des formations », La Lettre de I'OCIM [En ligne], 191 | 2020, mis en ligne le 01 septembre 2021, consulté le 12 décembre 2021. URL : http://journals.openedition.org/ ocim/4076; DOI : https://doi.org/10.4000/ocim.4076

Ce document a été généré automatiquement le 12 décembre 2021.

Tous droits réservés 


\section{Dans la jungle des formations}

\section{Serge Chaumier}

Atelier Visite au musée de l'Homme.

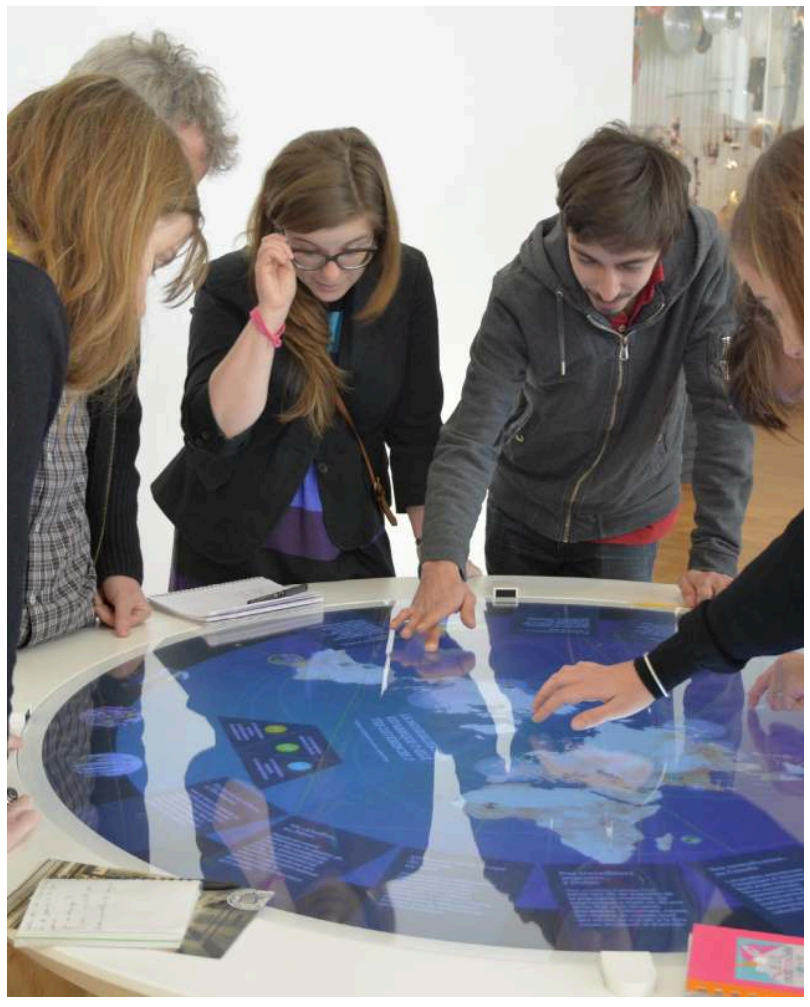

(c) L'Art de Muser/Master MEM

"Ce que nous devons apprendre à faire, nous l'apprenons en le faisant. »

2 Aristote

3 Se lancer dans le décryptage et la compréhension de l'offre de formations qui préparent aux métiers des musées demande une dose d'inconscience. Responsable de formation de master en muséologie-muséographie depuis vingt ans, et impliqué dans 
des formations depuis plus de trente ans, j'ai souvent moi-même beaucoup de mal à me repérer. Nous ne savons pas comment les étudiants peuvent s'y retrouver, pas davantage que les professionnels qui recrutent. Comment, dans un pays centralisé - qui entend contrôler, évaluer, autoriser - une telle cacophonie peut-elle perdurer ? Comment comprendre cette situation aussi pléthorique que globalement médiocre ? Nous avons conduit l'enquête en nous appuyant sur une étude spécifique et sur les données de l'observatoire de l'Ocim.

\section{Le contexte}

4 Cette étude porte exclusivement sur les formations de niveau master, elle ne prend pas en compte les diplômes de type licence professionnelle, ni même les diplômes d'université (DU), non reconnus officiellement. Nous ne nous intéressons qu'aux formations des universités et écoles associées, et laissons de côté l'offre conséquente portée par les écoles privées et instituts de formation professionnelle. Nous ne revenons pas sur les raisons historiques, ni sur la sociologie de l'université, qui expliquent l'explosion de l'offre de formation depuis le passage au LMD (licencemaster-doctorat) suite à la réforme dite « de Bologne » en $2004{ }^{1}$.

\section{Dénombrement et périmètre}

5 Pour les seules universités, nous avons dénombré un total de 114 formations qui affichent dans leur intitulé un lien avec le secteur des musées, du patrimoine, de la gestion de projets culturels ou de la médiation des arts ou des sciences. Nous avons retenu comme critère les formations qui indiquent dans leurs objectifs de professionnalisation, à un degré ou un autre, le secteur des musées et des expositions.

Car la délimitation n'est pas simple: des formations dont l'intitulé porte le terme de "patrimoine» avancent souvent l'objectif de former des futurs professionnels de musée. Les formations en médiation culturelle le revendiquent également - même si elles ne s'y limitent pas et peuvent être plus larges en préparant à des métiers de la médiation dans d'autres secteurs de la culture - de même que les formations à l'administration ou à la coordination de projets.

7 Ainsi, nous pouvons faire ce premier constat: considérant un nombre moyen de 20 diplômés par an et par formation, cela représente quelques 2280 jeunes diplômés censés s'insérer chaque année sur le marché du travail.

\section{Une cartographie des masters préparant aux métiers des musées}

Dans la continuité de l'observation réalisée en collaboration avec Icom France et les universités d'Avignon et d'Arras, l'Ocim vient de publier la troisième version de la cartographie interactive des masters préparant aux différents métiers des musées, du patrimoine et de la culture scientifiques et techniques. Au 1er avril 2020, cette cartographie recense les 64 formations ayant répondu à l'enquête menée en septembre 2018.

Conçue pour les étudiants et les professionnels en quête d'un master ou d'une formation continue, cette nouvelle version propose une interface dynamique de 
recherche. Elle permet notamment de repérer les formations les plus adaptées en combinant différents filtres : domaines thématiques, mots-clés, universités et/ou régions administratives. Il est également possible de limiter les résultats aux seules formations proposant l'accès en formation continue ou en apprentissage, proposant un stage, un mémoire et/ou des projets tuteurés. Une fiche descriptive, pour chacune d'entre elle, permet d'identifier les mentions disponibles, les parcours, la finalité et les coordonnées des masters.

- Pour figurer sur cette cartographie ou pour actualiser les données, merci de prendre contact avec l'Ocim : documentation.ocim@u-bourgogne.fr

- Consultable sur : https://utils.ocim.fr/cartomasters

Stéphane Chevalier

responsable du secteur

Information-Documentation de l'Ocim

Cartographie des masters préparant aux métiers des musées.

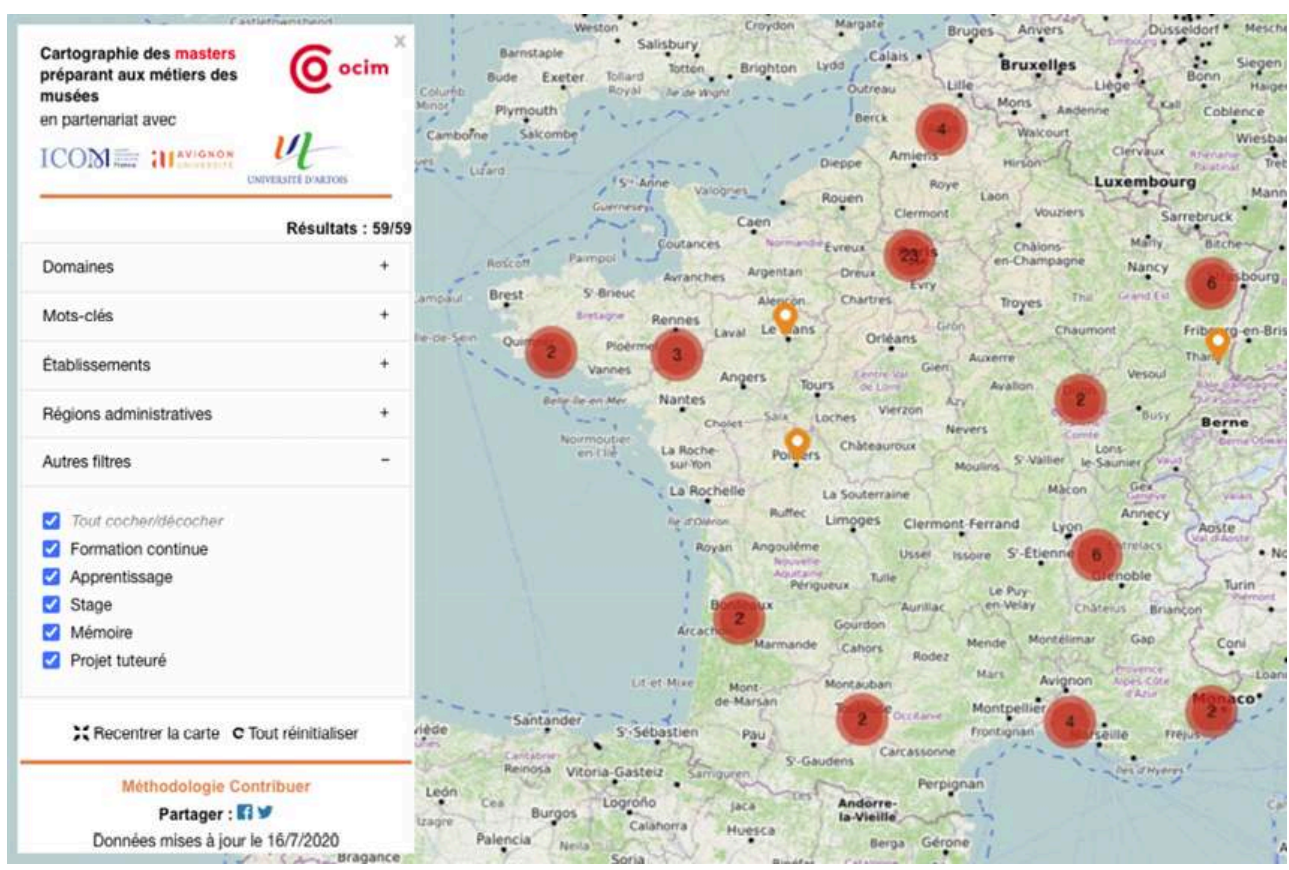

(c) Ocim

Ces 114 formations sont réparties dans 14 régions (soit presque toutes), et dans 69 institutions. En moyenne chaque université intègre donc presque deux formations qui visent des objectifs proches, mais souvent dans des départements différents et avec des nuances en matière de secteurs visés. Des formations dont les objectifs sont très ciblés, comme le commissariat d'exposition en art contemporain, le métier de régisseur ou la formation à la médiation scientifique ne ressortent pas des mêmes histoires et ne comportent pas les mêmes contenus. Malgré tout, les recherches d'économies qui sévissent dans l'université ont souvent pour effet de prôner des mutualisations et de favoriser les rapprochements - parfois enthousiastes et le plus souvent contraints - de 
cursus différents. Notons déjà ce choix initial de former de manière hyper spécialisée à des métiers très précis, ou au contraire de parier sur l'adaptabilité nécessaire et l'ouverture d'esprit avec une formation plus généraliste.

L'étude regroupe donc toutes les formations, des plus spécialisées (visant par exemple à former sur le numérique muséal ou encore des conseillers en ethnologie pour les projets culturels) aux formations qui appréhendent le secteur culturel comme un tout.

Mots-clés les plus utilisés dans les intitulés de diplômes des formations étudiées.

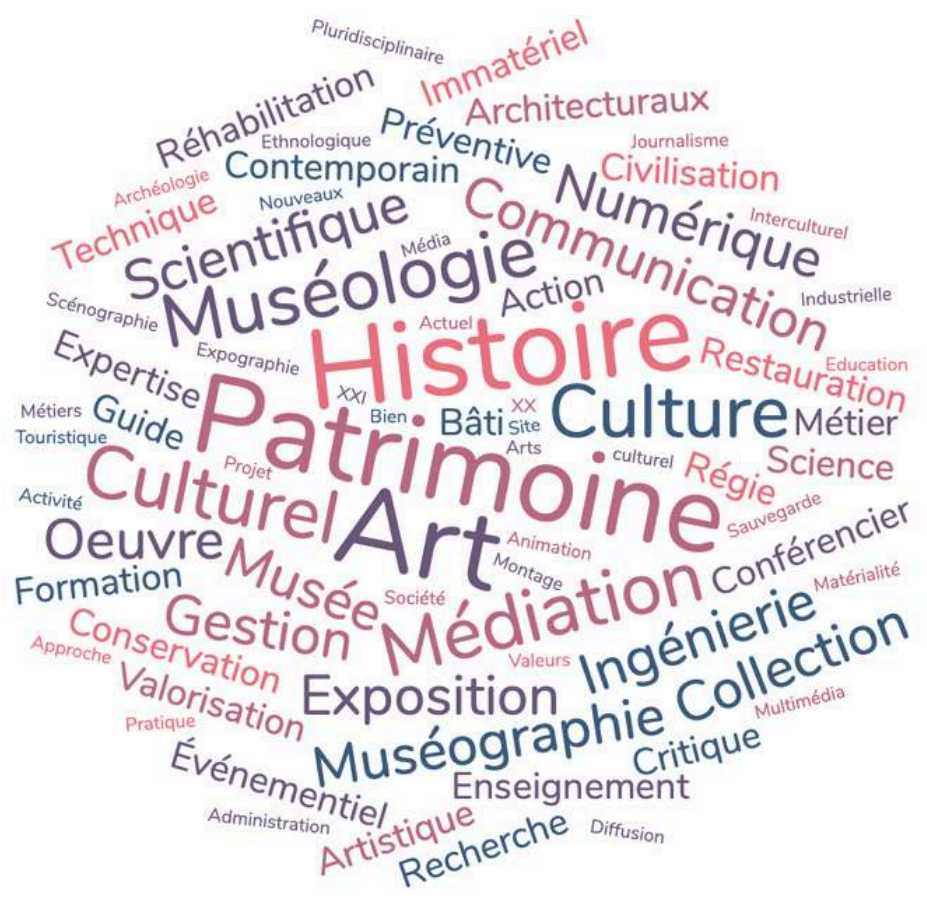

(C) DR

\section{Domaines}

Nous avons proposé aux responsables des formations référencées ${ }^{2}$ un questionnaire en ligne avec la possibilité de choisir deux domaines de prédilection caractérisant au mieux leur formation. Sur 18 mots proposés, ceux de "musée», "patrimoine», " histoire de l'art», "culture» et "culture scientifique» sont les plus plébiscités, comme le montre le nuage de mots précédent. Autre constatation, le domaine "science" attire $11 \%$ des réponses, s'attribuant ainsi une part non négligeable. De plus, et sans surprise, toutes les déclinaisons confondues du terme "patrimoine» totalisent $32 \%$.

11 Lorsqu'il est demandé de choisir les deux mots-clés les plus caractéristiques de la formation, la "médiation culturelle » arrive très largement en tête. Le terme «action culturelle $»^{3}$ plus précis historiquement que celui de "médiation culturelle» et supposant une conception plus engagée de la culture est plébiscité par moins de responsables. Enfin les contenus plus spécialisés et plus complexes tels que « expographie » ou « régie des collections » sont moins cités.

Si la plupart des masters développent une alternance entre cours et stages, il ne faut pas les confondre avec les masters en apprentissage où le jeune professionnel est 
salarié d'une institution et revient se former à l'université comme un salarié en formation continue. Seul six masters en France proposent actuellement la formation en apprentissage ${ }^{4}$.

\section{Volumes horaires}

13 Comparer les masters devient délicat lorsqu'on s'intéresse à leurs contenus et, en premier lieu, simplement aux heures dispensées. Près de la moitié des diplômes ne renseigne pas cet indicateur, ni en réponse à l'enquête, ni dans les informations glanées en complément sur leurs sites Internet. Souvent les plaquettes indiquent une liste de matières enseignées, mais sans volume horaire, alors même que les maquettes déposées au ministère pour accréditation des diplômes les rendent nécessairement disponibles. Pour la moitié répondant à cette question, le volume s'étend de 118 heures à 700 heures en master 1 (M1), avec une moyenne de 362 heures pour les 58 masters renseignés. Cela va de 104 à 448 heures pour les 49 masters 2 (M2) renseignés, soit une moyenne de 290 heures ${ }^{5}$. Ainsi, un même niveau de diplôme peut s'appliquer à une formation qui dispense quatre fois plus d'encadrement et d'enseignement qu'une autre, et ce sur une même année. Certaines formations sont à plein temps quand d'autres ne regroupent les étudiants qu'une journée ou deux par semaine.

\section{Contenus}

Les aspects concernant les contenus proposés - au-delà de vagues intitulés - et les approches pédagogiques suivies restent plus délicats à évaluer via les sites Internet ou le déclaratif en ligne des responsables de diplôme. Si on admet que la professionnalisation passe par autre chose que par la transmission de contenus excathedra en cours magistraux, il faut s'intéresser à des indicateurs plus fins. Par exemple, le nombre de participants dans une promotion, l'implication de professionnels, les partenariats avec les institutions, leur nature, la présence ou non de projets tuteurés et ce qu'ils signifient. Si les formations de préparation au concours de la Fonction publique peuvent légitimement mettre en avant l'intérêt de délivrer des contenus académiques, la formation aux métiers réels nécessite en revanche une mise en pratique pour transformer les connaissances en compétences. Les formations professionnelles supposent notamment de mettre les apprenants en situation de prouver leur capacité à conduire et à assumer des livrables professionnels. Il s'agit aussi d'être dans une relation de proximité avec le milieu visé et d'être présents sur le terrain, in situ. C'est pourquoi une formation réellement professionnalisante coûte cher et reste chronophage.

Nombre d'heures d'enseignement déclarées en 2e année de master (M2)

(c) S. Chaumier

Nombre d'heures relatives aux musées, cumulées sur les deux années du master (M1 + M2)

(c) S. Chaumier

La Lettre de l'OCIM, 191 | 2020 


\section{Sélections}

Seuls 34 masters indiquent recruter sur dossier plus entretien. Beaucoup de formations ne rencontrent pas les étudiants, qui consentent de moins en moins à parcourir la France au vu de leurs maigres finances. À ce sujet, il faudrait s'attarder sur les conditions de vie étudiante qui accroissent les inégalités entre ceux qui choisissent réellement leur formation et ceux qui postulent au sein de leur université, en restant chez leurs parents. La sélection pour entrer en M1 est variable... C'est parfois le nombre de places disponibles, ou le type de diplôme antérieur qui est déterminant, davantage que les expériences acquises ou le profil du candidat. Rares sont les formations qui insistent sur la créativité dans leurs critères d'admission.

Seules de petites promotions permettent un suivi personnalisé, mais aussi de conduire des projets grandeur nature et de se déplacer sur le terrain. Si les effectifs des spécialités en master 2 (M2) sont en grande majorité compris entre 15 et 25 étudiants, certaines formations mutualisent parfois un tronc commun entre plusieurs spécialités. Mais c'est surtout en master 1 que les mutualisations sont quasi-systématiques, souvent pour des raisons économiques. On s'aperçoit que jusqu'à 8 spécialités peuvent être regroupées sur des cours communs, soit jusqu'à 200 étudiants réunis! Certaines spécialités - qui ont des difficultés à recruter et remplir la promotion - peuvent être regroupées pour les $2 / 3$ de la formation dispensée. On peut estimer qu'il y a parfois un écart avec l'offre annoncée quand une spécialité est noyée parmi 3, 4, 5, 6, 7, voire 8 autres!

17 Un des critères analysés est celui des objectifs métiers autoproclamés par la plupart des formations. Certaines n'hésitent pas à promettre toute une panoplie de postes de direction et à avancer des métiers sans aucun rapport avec les contenus annoncés de la formation. Par exemple, les métiers d'administrateur ou de coordonnateur de projet peuvent être indiqués, en l'absence de toute notion de gestion parmi les cours dispensés... Nous relevons parfois des perles, comme ce diplôme qui prétend former des " cadres en cabinet de muséographie "! La même formation indique aussi "animateur du patrimoine", "médiateur» ou "chargé de communication» ou encore " enseignant-chercheur ». Plus rares sont les formations qui ciblent un domaine précis, avec plus de cohérence dans le contenu. C'est notamment le cas pour les métiers de régisseur, médiateur numérique ou webmaster, qui semblent avoir le vent en poupe. Ce critère des objectifs et des métiers visés est pourtant celui qui est principalement regardé par les postulants. Quant au taux d'insertion, ce sont des déclaratifs qui en général annoncent entre 80 et $97 \%$, sans en apporter la moindre preuve. Très peu de formations présentent les annuaires des situations réelles des diplômés.

\section{Intervenants}

Le nombre de statutaires universitaires varie de 2 à 45 . En moyenne, ils sont souvent entre 10 et 20 à intervenir dans le cursus. Concernant le nombre de professionnels et la place qu'ils occupent dans la formation, les réponses fluctuent entre 2 et 100 intervenants. Le plus souvent une quinzaine. Il faudrait voir plus finement si ces derniers sont impliqués au-delà du caractère honorifique d'intervenir à l'université. Cela suppose que les professionnels puissent apporter un véritable encadrement sur les 
travaux conduits pour établir un compagnonnage en direction d'une véritable insertion.

\section{Stages}

19 La plupart des formations incluent un stage obligatoire. Si ce dernier est en moyenne de trois mois, de plus en plus de formations proposent une durée de deux fois deux mois, puisque les institutions doivent légalement gratifier les stages au-delà de cette période. Certains masters, dits professionnels, annoncent un stage de 6 semaines en M1 (et même parfois de 15 jours) et seulement de deux mois en M2. Au vu du nombre de formations et d'étudiants en recherche de stages, nous comprenons que la situation soit saturée.

\section{Contenus dispensés}

Ceux-ci varient en fonction des orientations de la formation, mais souvent aussi en fonction des domaines de spécialité et des centres d'intérêt des enseignants statutaires. C'est un des problèmes de l'enseignement supérieur que d'avoir mis le cap sur la professionnalisation sans disposer bien souvent des personnels compétents pour cela : d'où la présence indispensable de professionnels au sein des cursus. Si nous nous attardons sur les modules en muséologie ou muséographie, il s'agit le plus souvent d'un enseignement en patrimoine qui inclut l'histoire des musées. Comment peut-on former à des métiers spécifiques, comme la conception d'exposition, avec 24 ou même 40 heures sur deux ans?

\section{Muséologie}

Pour établir le graphique ci-dessus - et construire une représentation objective - nous n'avons retenu, en consultant les maquettes présentes sur les sites internet des formations, que les enseignements qui concernent explicitement les musées (muséologie, muséographie, histoire ou gestion des musées, sémiotique de l'exposition, scénographie), en excluant les thématiques sur les publics, la médiation ou la gestion de projet, qui peuvent ne pas être appliquées aux seuls musées. Il apparait qu'une majorité de masters (soit 31 masters) propose un volume horaire de moins de 100 heures sur deux ans en muséologie et seulement 17 plus de 100 heures $^{6}$. Nous pouvons raisonnablement penser que seules ces formations, avec toutes leurs différences, sont réellement des formations professionnelles pour les musées.

\section{Mémoires et projets tutorés}

La plupart des formations exigent de rédiger et soutenir un mémoire. La volonté d'expérimenter d'autres formes est rarement de mise, pourtant il n'est pas certain que le mémoire soit la forme la plus adaptée. Certes, il impose de faire des recherches, de les synthétiser, de problématiser, de conduire parfois une enquête, de savoir rédiger, et enfin de soutenir devant un jury: toutes des compétences utiles, mais que l'on peut acquérir par d'autres voies. 47 formations déclarent mettre en place des projets 
tutorés. Cette évolution recouvre toutefois une grande hétérogénéité dans la réalité des projets conduits, et surtout du suivi réalisé ${ }^{7}$. Cela peut prendre la forme d'une action courte, anecdotique dans le diplôme, ou à l'inverse d'un projet structurant sur les deux années du cursus. Cela peut avoir des formes assez simples, comme la réalisation d'un événement, l'animation de la Nuit des musées ou d'une Nuit des étudiants, l'organisation d'une journée d'étude, voire d'un séminaire, la réalisation d'une étude fictive, ou encore d'un workshop. Parfois, c'est l'écriture de critiques pour alimenter un blog, ou la conception de médiation, de fiches de salle, de réalisation de visites guidées. Toutes choses que d'autres masters pratiquent couramment, sans pour autant le caractériser de « projets tuteurés».

D'autres citent l'organisation d'un voyage d'étude par les étudiants. Plus conséquemment, cela prend la forme de la réalisation d'un diagnostic, d'un audit, d'une étude de faisabilité, ou de connaissance des publics, d'une évaluation d'exposition. Plus rarement cela s'incarne dans un chantier des collections ou encore par la réalisation d'une exposition grandeur nature.

Seules 38 formations précisent ce que sont réellement les projets tuteurés mis en œuvre et parmi les formations qui affirment préparer aux métiers des musées, seules 7 formations réalisent réellement des expositions. Dans ce dernier cas, cela peut concerner autant l'accrochage de travaux d'un artiste dans un hall ou une galerie de l'université que la réalisation de vraies expositions construites en partenariat au sein d'une institution. Les budgets de ces opérations vont de 800 à 250000 euros. 33 responsables de formation indiquent le nom des partenaires institutionnels avec lesquels les coopérations sont conduites. Il faudrait pouvoir entrer dans le détail pour mieux comprendre les mécanismes de ces partenariats : parfois ceux-ci consistent en une sortie sur site (pour rencontrer les professionnels dans leurs murs) ou participer à une journée d'étude. Dans la plupart des cas, ce sont des partenariats locaux, parfois pour un événement régulier et en quelque sorte institué dans la formation. Plus rares sont les formations qui développent pour la réalisation des projets tuteurés des partenariats avec les institutions culturelles sur le long terme.

La consultation des sites Internet est assez instructive. Le plus souvent peu de choses sont indiquées et, à l'inverse, beaucoup plus rarement, il est difficile de ne pas perdre l'interlocuteur dans la multiplicité des actions. À ce sujet, il est étonnant que si peu de formations disposent de sites Internet dédiés, et encore moins d'une véritable politique d'animation sur les réseaux sociaux. Seule une petite dizaine de formations se montre réellement active. D'autres se sont lancées dans la tenue d'un journal ou d'un blog en ligne, ce qui suppose un investissement important des responsables de diplômes pour faire tenir l'initiative dans la durée.

Nous comptons ainsi 18 sites Internet dédiés : les contenus sont souvent peu détaillés sur la formation; parfois difficiles à trouver, il faut télécharger le guide des études en PDF pour disposer des listes de cours, avec (pas toujours) les volumes horaires. Les contenus sont mis à jour tous les 4 ans, lors du renouvellement des maquettes, ou quand un nouveau responsable de diplôme s'y attèle.

\section{Conclusion}

Rappelons aux professionnels leur part de responsabilité, et aussi leurs intérêts à regarder de près les contenus des formations, que ce soit lorsqu'ils recrutent, un 
stagiaire, un apprenti ou un employé. Depuis peu, il n'y a plus officiellement de sélection entre le $\mathrm{M} 1$ et le $\mathrm{M} 2$, ce qui ne fait qu'aggraver une situation déjà dramatique. Car ce n'est pas le fait d'avoir ou non un master 2 qui fait la différence entre les candidats à un emploi. Beaucoup d'étudiants obtiennent un titre de master avec, comme nous l'avons montré dans cet article, une forte hétérogénéité entre de bons et de mauvais diplômes. Ce qui compte, ce ne sont ni les notes, ni les titres, mais ce que l'étudiant a fait véritablement pendant la formation.

\section{BIBLIOGRAPHIE}

Chaumier S. Réflexions sur les stages et les conditions d'accueil d'un stagiaire dans une institution culturelle, la lettre de l’ocim, n¹52, mars-avril 2014, pp. 5-13.

Chaumier S. Nouveauté dans les musées et lieux d'exposition, les apprentis en muséographie, in la lettre de l'Ocim, $\mathrm{n}^{\circ} 176$, mars-avril 2018, pp. 12-16.

\section{NOTES}

1. Chaumier S. Les formations aux métiers de la culture : de la génération spontanée au désir de régulation. U-culture(s) : revue culturelle annuelle de l'université de Bourgogne, ${ }^{\circ} 1,2006$.

2. Voir la cartographie des masters préparant aux métiers des musées, Ocim : https:// utils.ocim.fr/cartomasters

3. Chaumier S. et Mairesse F. La Médiation culturelle. Paris : Armand Colin, 2018, 2de édition, 280 p.

4. Pour faire exception, puisque nous ne mentionnons pas dans cet article de noms de formations, rappelons que le master Expographie-Muséographie de l'université d'Artois a été le premier, depuis 2015, dans le secteur des musées à proposer l'apprentissage et demeure encore l'un des rares à avoir la promotion complète salariée en institution.

5. Ces données sont à manier avec précautions, car elles incluent parfois des heures de stage ou de direction de mémoire. Pour envisager la formation, il faudrait additionner sur le cycle complet, M1 et M2 (de 160 à 934 heures, soit 530 heures en moyenne sur 64 répondants), or certains diplômes ne dispensent leur spécialité qu'en M2, et parfois sur un seul semestre, de septembre à décembre, avant un départ en stage. Les volumes horaires les moins élevés traduisent les anciens masters re-cherche (ex-DEA), alors que les plus pourvus traduisent des formations professionnalisantes (ex-DESS).

6. Parmi les 17 formations, il y a parfois des parcours différents au sein d'un même master, à l'exemple de l'École du Louvre qui représente 4 parcours parmi les 17 inventoriés.

7. Les projets tuteurés peuvent être ou non inclus dans les maquettes en heures étudiants. 


\section{RÉSUMÉS}

Nombre d'heures dispensées, contenus des enseignements, activités professionnalisantes, sélection à l'entrée et spécialisations... L'auteur passe au crible les formations aux métiers des musées, en s'appuyant sur une observation menée sur l'offre de masters en France.

\section{INDEX}

Mots-clés : formation professionnelle ; métier/personnel des musées ; organisation de l'enseignement supérieur; musée général

\section{AUTEUR}

\section{SERGE CHAUMIER}

Professeur et responsable du master Expographie-Muséographie à l'université d'Artois serge.chaumier@univ-artois.fr 